\title{
Studies on Biotinylated Liposomes as Potential Carries for Enhancing Oral Absorption of Curcumin
}

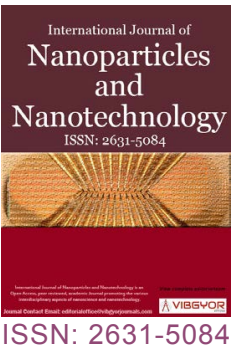

\section{Qiuping Wu $u^{1}$, Kunquan Su', Jin Tao', Zeng Tan ${ }^{1}$, Chenyan $W u^{2}$, Lingzhi yin ${ }^{3}$ and Ying $H u^{1^{*}}$}

'Zhejiang Pharmaceutical College, Zhejiang, China

${ }^{2}$ Dongyang People's Hospital, Zhejiang, China

${ }^{3}$ Tigermed Consulting Co., Ltd, Zhejiang, China

\begin{abstract}
Oral dosage forms have many obvious advantages like simple, painless and self-administered. While the bioavailability of drugs are always low for they are unstable and usually degraded within gastrointestinal tract. Curcumin, a multifunction native compound, can be used in curing many diseases while the oral bioavailability is relatively low, limiting its clinical effectiveness. The aim of this study was to investigate the method of increasing the bioavailability by the addition of biotin, which was a kind of vitamin helping the liposomes to uptake by biotin receptors. In situ rat Single-Pass Intestinal Perfusion Model (SPIP) was applied to investigate the different content of biotin and caco- 2 cells monolayer model was established to mimic the intestinal tract in drug transportation study. As the results shows that biotin decorated liposomes (biotin-PEG-DSPE-Lip) had a better cellular uptake and transportation rate compared to biotin undecorated ones (PEG-DSPE-Lip). It indicated that the bioavailability of curcumin had been increased.
\end{abstract}

\section{Keywords}

Curcumin, Biotinylated, Liposomes, Oral, Caco-2 cells

\section{Introduction}

Curcumin (Cur) is a kind of polyphenols natural active component extracted from rhizoma curcumae longae, having a rich application in food processing because of its inexpensive and oral security [1]. Modern research has found other uses for Cur, such as antitumor [2], antioxidant, anti-inflammatory [3], cardiovascular therapy and neurodegenerative pathology [4]. However, it has poorly sol- ubility in water and scarcely stable in physiological environment because of alkaline condition [5]. Otherwise, it would be removed rapidly from body after administration. So the bioavailability of curcumin has been seriously limited.

Liposomal strategy is a method to create a cytomembrane-like structure to increase the drug delivery and utilization $[6,7]$. Liposomes have been widely used for their safety and multifunction when

*Corresponding author: Ying Hu, Zhejiang Pharmaceutical College. No. 888, East Section, Yinxian Main Road, The Zone of Higher Education, Ningbo, Zhejiang, 315100, China

Accepted: March 14, 2020; Published: March 16, 2020

Copyright: (C) 2020 Wu Q, et al. This is an open-access article distributed under the terms of the Creative Commons Attribution License, which permits unrestricted use, distribution, and reproduction in any medium, provided the original author and source are credited.

Wu et al. Int J Nanoparticles Nanotech 2020, 6:034 
they are decorated by appropriate ornament. For the purpose of improving the oral bioavailability of curcumin, Ana Catalan-Latorre [8] combined phospholipid, Eudragit ${ }^{\circledast}$ S100 and hyaluronan sodium salt to obtain freeze-dried eudragit-hyaluronan immobilized liposomes. It demonstrated much more stable in gastro-intestinal tract condition and had an increase in caco- 2 cell viability. Physiological obstacles have precluded much oral administration of liposomes, so in order to increase intestinal uptake, liposomes can be conjugated with various ligands. Ying Li [9] did research on DSPE-PEG-cholic acid modified liposomes (CA-LPs), trying to elucidate the mechanisms of cholic acid receptor-mediated uptake to hepatic delivery. CA-LPs had the highest transport across caco- 2 cells and highest cellular uptake by HepG2 cells.

Biotin (Bio) also named vitamin $\mathrm{H}$, is a kind of water-soluble vitamins [10]. Bio plays significant role in synthesizing vitamin $\mathrm{C}$, also taking participate in metabolism of fat and protein. While our body can't produce Bio that just depends on food intake or synthesis by intestinal flora. The specific nature of Bio decides multifunction in oral administration in human body [11]. Delia Mandracchia [12] synthesized and evaluated biotin-decorated polymathic INVITE micelles, which revealed a faster transport across caco- 2 cells monolayer than the undecorated micelles, and the biotin-decorated micelles enhanced drug accumulation in tumor-cells that over expressing receptor for biotin.

The purpose of our study is to enhance the cellular transport of cur liposomes. We choose Bio-PEGDSPE decorated and DSPE-PEG decorated lectins to prepare Biotin decorated and undecorated liposomes. What we hope to accomplish is to find out that biotin can help liposomes to transport across the intestinal tract, thus improving the oral bioavailability of curcumin. Caco- 2 cells are implied to establish the cell model, as they are tumor-derived cells that can be differentiated to monolayer and simulate the intestinal tract. Furthermore, in situ single-pass intestinal perfusion (SPIP) model also used in the study, to determine the percentage of Bio-PEG-DSPE in lectins.

\section{Materials and Methods}

\section{Materials}

Curcumin ( $97 \%$ in purity) was purchased from Mellon biological co., LTD (Dalian, China). Lecithin was purchased from Bo'ao Biological Technology Co., Ltd. (Shanghai, China). Cholesterol was purchased from Wuhan life technology co., LTD (Wuhan, China). DSPE-PEG ${ }_{2000}$ lecithin and Biotin-PEG ${ }_{2000}$-DSPE lecithin were purchased from Ponsurebio technology co., LTD (Shanghai, China). Male SD rats were obtained from Zhejiang academy of medical sciences of laboratory animal center (Hangzhou, China). Caco-2 cell line was received from Shanghai Institute of Materia Medica (Shanghai, China). Fetal Bovine Serum was purchased from HyClone Company (Logan, Utah, USA).

\section{Preparation of oral curcumin liposomes}

Three types of oral curcumin liposomes (N-Lip; DSPE-PEG-Lip; Biotin-PEG-DSPE-Lip) were formulated by film-ultrasonic technique [13]. Briefly, lipids and Cur (Table 1) were dissolved in chloroform respectively to obtain solutions. Removing the chloroform and forming a thin film on the inner wall of the flask via vacuum rotary evaporation. Glucose solutions $(5 \%, 10 \mathrm{ml}, \mathrm{w} / \mathrm{w})$ were used to dissolve the film by shaking and hydration for $30 \mathrm{~min}$. The solution was treated with ultrasonic ( $100 \mathrm{w}, 5 \mathrm{~min})$ and filtered through the $0.22 \mu \mathrm{m}$ membrane filter to get the liposomes.

\section{Characterization of oral curcumin liposomes}

Size, polydispersity and zeta potential: Three types of liposomes were diluted to appropriate concentration before measuring the size, polydispersity (PDI) and zeta potential. All determinations were recorded at room temperature $\left(25^{\circ} \mathrm{C}\right)$ using a Zetasizer Nano ZS90 (Malvern Instruments, Malvern, UK).

Morphology of the liposomes: Scanning Electron Microscope (SEM) was used to analyze the

Table 1: Liposomes compositions used in the study.

\begin{tabular}{|l|l|l|l|}
\hline Liposome type & Lecithin type & Dosage (mg) & Cur (mg) \\
\hline N-Lip & Injectable lecithin & 100 & 5 \\
\hline DSPE-PEG-Lip & DSPE-PEG & 100 & 5 \\
\hline Biotin-PEG-DSPE-Lip & Biotin-PEG-DSPE & 100 & 5 \\
\hline
\end{tabular}


morphology of cur liposomes. (SEM, Leo DSM982 Gemini, LEO Electron Microscopy Inc., Oberkochen, Germany). Samples were prepared onto copper grids (Agar Scientific Ltd., Essex, England) or onto copper grids coated with carbon film (Agar Scientific Ltd., Essex, England) either directly from gas-borne particles using an electrostatic point-toplane precipitator or from collected dry powders by gently dipping the sample grid into the powder and carefully blowing off excess material.

Loading and entrapment efficiency: The entrapment efficiency (EE\%) of the liposomes were determined by destroying the liposomes with Triton $X-100$, then measuring the concentration of cur by HPLC method. A Sephadex-G50 chromatography was used to separate the free drug and liposomes. EE\% was calculated by the following equation $[14,15]$ :

$$
E E \%=\frac{M_{\text {Tot }}-M_{\text {Free }}}{M_{\text {Tot }}} \times 100 \%
$$

\section{Optimization of biotinylation in oral curcumin liposomes}

Stability in intestinal fluid: Before investigating the intestinal perfusion of liposomes, the stability in intestinal fluid of cur was studied. Incubating the $\mathrm{K}-\mathrm{R}$ solution [16] in $37{ }^{\circ} \mathrm{C}$ and equilibrating the intestinal tract at a constant rate of $2 \mathrm{ml} / \mathrm{min}$ before collecting the intestinal fluid. Then preparing the Cur- intestinal fluid solution at concentrations of $20,40,80 \mu \mathrm{g} / \mathrm{ml}$, and sampling at $0,0.5,1,1.5,2$, $2.5 \mathrm{~h}$ followed the HPLC determination.

\section{Rat single-pass intestinal perfusion}

Animals: Male and female Sprague-Dawley (SD) (200 250 g) rats were supplied by Zhejiang Medical Academy (Hangzhou, China). The rats were housed under controlled conditions of temperature $(25 \pm 2$ $\left.{ }^{\circ} \mathrm{C}\right)$ and relative humidity $(50 \% \pm 20 \%)$, acclimating for one week and fed a standard diet and water. Before experiment, the rats were fasted for $12 \mathrm{~h}$ with free access to water. All animal experiments were conducted in accordance with the Guidelines of current ethical regulations for institutional animal care and use by Zhejiang Medical Academy.

The SPIP study was performed as the previously reported methods $[17,18]$. Briefly, rats were anesthetized by intraperitoneal (i.p.) injection of $20 \%$ urethane $(1.0 \mathrm{~g} / \mathrm{kg})$, then abdomen were opened along the medioventral line and a duodenal seg- ment $(10 \mathrm{~cm})$ was isolated and ligatured with silk ligature. Rats were kept warm and moisturized throughout the whole experiment. All solutions were incubated in a $37{ }^{\circ} \mathrm{C}$ water bath.

Before starting perfusion, the isolated segment was rinsed with blank Krebs-Ringer's buffer for approximately $30 \mathrm{~min}$. Then, they were perfused with test liposomes-containing Krebs-Ringer's perfusate at $37^{\circ} \mathrm{C}$ at $0.2 \mathrm{ml} / \mathrm{min}$ for $30 \mathrm{~min}$ to reach a steadystate. Samples were collected at each $15 \mathrm{~min}$ intervals in EP tubes throughout the whole collection and weighted before stored at $-80^{\circ} \mathrm{C}$ until analysis. The length and width of each segment were accurately measured at the end of the experiment.

The Ka of curcumin liposomes in the SPIP study can be calculated from the following equation [19]:

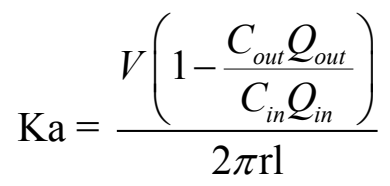

Where $\mathrm{V}$ is the perfusion rate of the liquid; $C_{\text {in }}$ and $C_{\text {out }}$ are the concentration of Cur in the inlet and outlet perfusate; $Q_{\text {in }}$ and $Q_{\text {out }}$ are the volume of entrance and exit intestinal perfusate; $R$ and $I$ are radius and length of the intestinal segment.

\section{In vitro release of curcumin from liposomes}

The in vitro release of cur from free drug and three types of cur liposome formulations were determined by dialysis. Briefly, $5 \mathrm{ml}$ samples in the dialysis bags were placed into $15 \mathrm{ml}$ dissolve medium containing phosphate buffer $(\mathrm{pH}=5.8)$ and ethanol solution (30\%) in conical flasks. Release condition was chosen $37{ }^{\circ} \mathrm{C}$ shaking bath with $200 \mathrm{r} / \mathrm{min}$ stirring. At $0.5,1,1.5,2,2.5,3,4,5,6,8,10,12$, 24, $36,48,60,72,96 \mathrm{~h}, 1 \mathrm{ml}$ liquid were sampled followed by filtration through $0.45 \mu \mathrm{m}$ membrane and analysis with high-performance liquid chromatography (HPLC). $1 \mathrm{ml}$ new dissolve medium were replenished to the flasks. Free curcumin solution was taken as the control experiment.

\section{Determination of in vitro absorption mecha- nism}

Cell viability study (MTT assay): Caco-2 cells were grown as monolayer in T25 cell culture bottles, at $37{ }^{\circ} \mathrm{C}$ in humidified atmosphere of $5 \% \mathrm{CO}_{2}$ in air. Dulbecco's Modified Eagle Medium (DMEM) high glucose, containing $1 \%$ nonessential amino acids, supplemented with $10 \%$ fetal bovine serum, 
$1 \%$ streptomycin and penicillin, was used as growth medium. Cells were seeded into 96 -well plates at a density of $1 \times 10^{5}$ cells/well [20]. After $24 \mathrm{~h}$ incubation, the cells were treated with $100 \mu$ of different types of cur liposomes, diluted with growth medium corresponding to the following concentrations: $300,150,75,37.5,18.75,9.375 \mu \mathrm{g} / \mathrm{ml}$ of cur. After $24 \mathrm{~h}$, the cells were washed three times with fresh medium, and the viability was determined by the MTT assay by adding $20 \mu \mathrm{M}$ MTT reagent to each well. After $4 \mathrm{~h}$, the formazan crystals were dissolved in $150 \mu \mathrm{l}$ dimethyl sulfoxide (DMSO), and their concentration was spectrophotometrically quantified at $490 \mathrm{~nm}$ with a microplate reader (Thermo Fish Scientific, America). Each concentration was repeated six wells. Results were shown as the percentage of non-treated control cells $(100 \%$ viability).

\section{Establish the caco-2 monolayer model}

TEER value: Cells were seeded into a transwell chamber at a density of $1 \times 10^{5}$ cells $/ \mathrm{ml}$, with 0.2 $\mathrm{ml}$ and $0.8 \mathrm{ml}$ culture solution in AP (upper space) and $\mathrm{BL}$ (down space) relatively, cultivating for nearly 21 days [21-23]. Before employing cellular uptake experiment, the caco- 2 monolayer should be confirmed to make sure the monolayer has been tight enough, the TEER value on both sides of the monolayer had been measured by a Millicell ERS resistance instrument (Millipore, America).

Alkaline Phosphatase Activities: ALP/AKP kit was used to investigate the activities of monolayer and evaluate the differentiation of caco-2 cells. Culture medium in AP and BL was suck out carefully and operate as the kit specification described.

\section{Permeability assessment of the Cur liposomes}

The caco-2 monolayer was washed by Hank's buffer solution in triplicate, and the Cur liposomes were preheated to $37{ }^{\circ} \mathrm{C}$ before added into the transwell plates, where $0.2 \mathrm{ml}$ samples in AP sides and $0.8 \mathrm{ml}$ free Hank's in BL sides [24-26] Sampling $0.1 \mathrm{ml}$ receptor fluid from the $\mathrm{BL}$ side separately at $30,60,90,120 \mathrm{~min}$ and supplemented with fresh Hank's buffer in the same volume. HPLC analysis the Cur concentrations and the final $\mathrm{P}_{\text {app }}$ data was calculated by the follow equation:

$$
P_{a p p}=\frac{d Q}{d t} \cdot \frac{1}{A C_{0}}
$$

Where $A$ is the permeable membrane area $(0.3$ $\left.\mathrm{cm}^{2}\right) ; C_{0}$ is the initial concentration of Cur in AP side $(\mu \mathrm{g} / \mathrm{ml}) ; \mathrm{dQ} / \mathrm{dt}$ is the diffusion flux. All the results were listed as mean \pm SD.

\section{Caco-2 cells uptake study}

The uptake study of curcumin liposomes were assayed with flow cytometry (BD FACS Calibur, America). Before detection, caco- 2 cells were planted in 24 well-plate with $5 \times 10^{4}$ cells/well and cultivated for almost 14 days. Then cells were washed with Hank's buffer for three times and treated with 0.5 $\mathrm{ml}$ liposomes (as curcumin concentration of 4, 8, $16 \mu \mathrm{g} / \mathrm{ml}$ ). Following 1 hour cultivation, liposomes were discarded and cells were detected.

\section{Statistical Analysis}

All experiments were performed in triplicate. All mean values are presented as mean \pm SD. Statistical analysis was performed by Prism Statistics 7.0 at a significance level of $P \leq 0.05$ and dramatic significant level of $P \leq 0.01$.

\section{Results and Discussion}

\section{Characterization of Cur liposomes}

In our study, film dispersion method was employed to prepare Cur liposomes because of its high encapsulation efficiency and stability as we
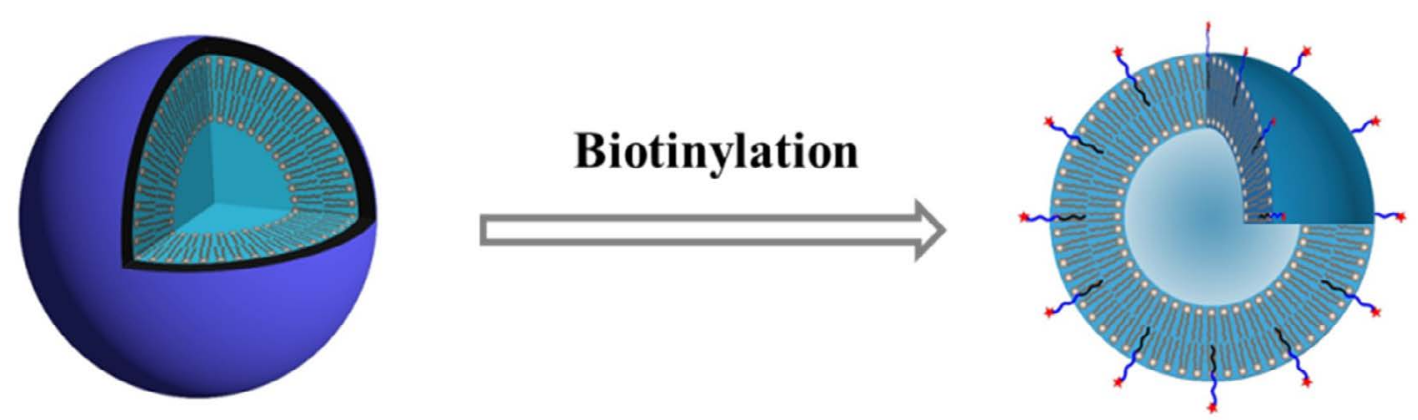

Figure 1: The diagram of normal liposomes and biotinylation liposomes. 
Table 2: Stability of cur in intestinal tract between $2.5 \mathrm{~h}$.

\begin{tabular}{|l|l|l|l|l|l|}
\hline Conc. $(\mu \mathrm{g} / \mathrm{ml})$ & \multicolumn{5}{|c|}{ Remaining percentage } \\
\cline { 2 - 6 } & $0.5 \mathrm{~h}$ & $1 \mathrm{~h}$ & $1.5 \mathrm{~h}$ & $2 \mathrm{~h}$ & $2.5 \mathrm{~h}$ \\
\hline 20 & 99.98 & 99.46 & 99.37 & 98.74 & 98.02 \\
\hline 40 & 99.69 & 99.72 & 99.21 & 98.48 & 98.04 \\
\hline 80 & 99.52 & 99.47 & 99.03 & 98.61 & 98.11 \\
\hline
\end{tabular}

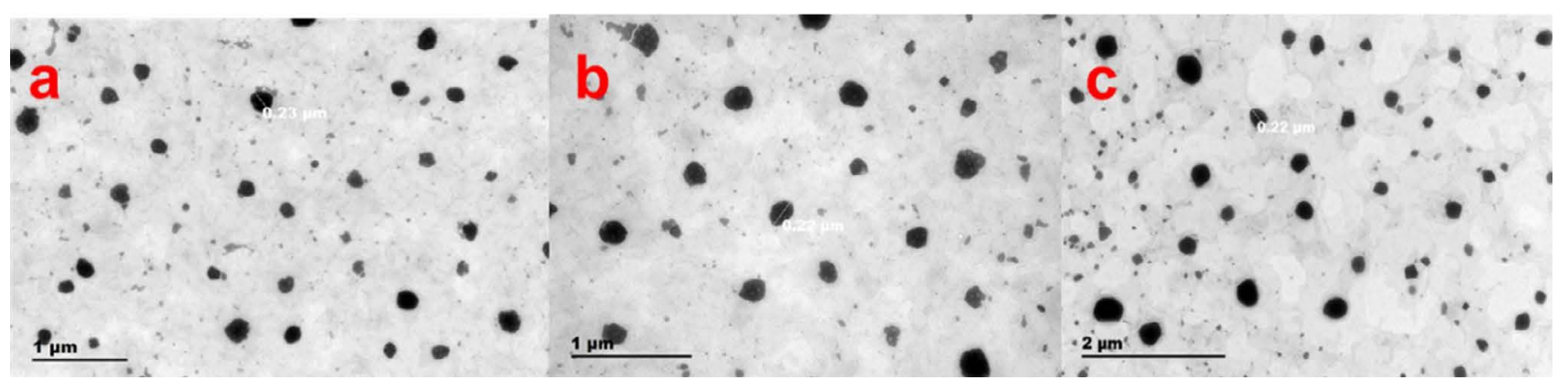

Figure 2: Morphology of the Cur liposomes. a) Morphological of N-Lip; b) Morphological of PEG-DSPE-Lip; c) Morphological of Biotin-PEG-DSPE-Lip.

studied previously. The EE\% of common Cur liposomes ( $\mathrm{N}$-Lip) was $81.5 \% \pm 2.17 \%$, almost the same after adding DSPE-PEG (DSPE-PEG-Lip) and BioPEG-DSPE (Biotin-PEG-DSPE-Lip). As show in Figure 1 , the surficial morphology of Cur liposomes were smooth and rounding, with the particle size in average value of 204.3, 208.3 and $212.3 \mathrm{~nm}$, respectively. Moreover, Zeta potential were determined as $-28.0,-24.4$ and $-21.2 \mathrm{mV}$, which reflecting the surface charge between particles, indicating the structures of the liposomes were relatively stable.

\section{Optimization of Biotin-PEG-DSPE-Lip}

The stability of curcumin in intestinal fluid was studied see Table 2. Curcumin is instability in alkaline environment, and oral liposomes contacted the gastrointestinal tract directly. Liposomes would have various behaviours as different ratios of biotin in lectin, thus, it is needful to investigate the content of biotin in lectin, which we chosed three biotin percentage about $5 \%, 7 \%, 10 \%$, as the same to PEG content. As Figure 2 shows that biotin combined liposomes had higher transport rate than discombined ones. As the content of PEG increased, less cur were crossed the intestinal tract, indicating that PEG hydrophilic composition, which can hinder the liposomes absorpted. Otherwise more PEG would cause the unstable of liposomes, consequently, less cur were transported. As to BiotinPEG-DSPE-Lip, the highest transport rate appeared on $7 \%$ biotin liposomes, higher biotin concentration may lead to instability caused by the excessive biotin which is also hydrophilic as the PEG. So we selected $7 \%$ biotin-PEG-DSPE-Lip as the biotin decorated liposomes, compared to the undecorated ones, improving the function of biotin on promoting drug transportation across the intestinal tract.

\section{In vitro release of Cur liposomes}

The in vitro release of free curumin and three kinds of Cur liposomes were compared in $48 \mathrm{~h}$ (Figure 3), which free drug curcumin had been almost released in $48 \mathrm{~h}(96.64 \%), \mathrm{N}$-Lip had a slower release about $69.48 \%$ in $28 \mathrm{~h}$, while the DSPE-PEG-Lip and Biotin-PEG-DSPE-Lip just released $35.42 \%$ and $33.47 \%$ respectively during the same time. Compared to free drug, Cur liposomes displayed obvious sustained-release effect, which can be long-circulated after absorbed into blood owing to PEG added. It is very important for curcumin to cure cancer by oral administration.

\section{In vitro absorption mechanism}

MTT assay compared to the normal liposomes and PEG decorated liposomes, biotin decorated liposomes showed the almost cytotoxicity, which the IC50 reached to $140.23 \mu \mathrm{g} / \mathrm{ml}$ (Figure 4), what's meaning that adding biotin didn't decrease the effect of curcumin.

Caco-2 monolayer model: Caco-2 cells derived 


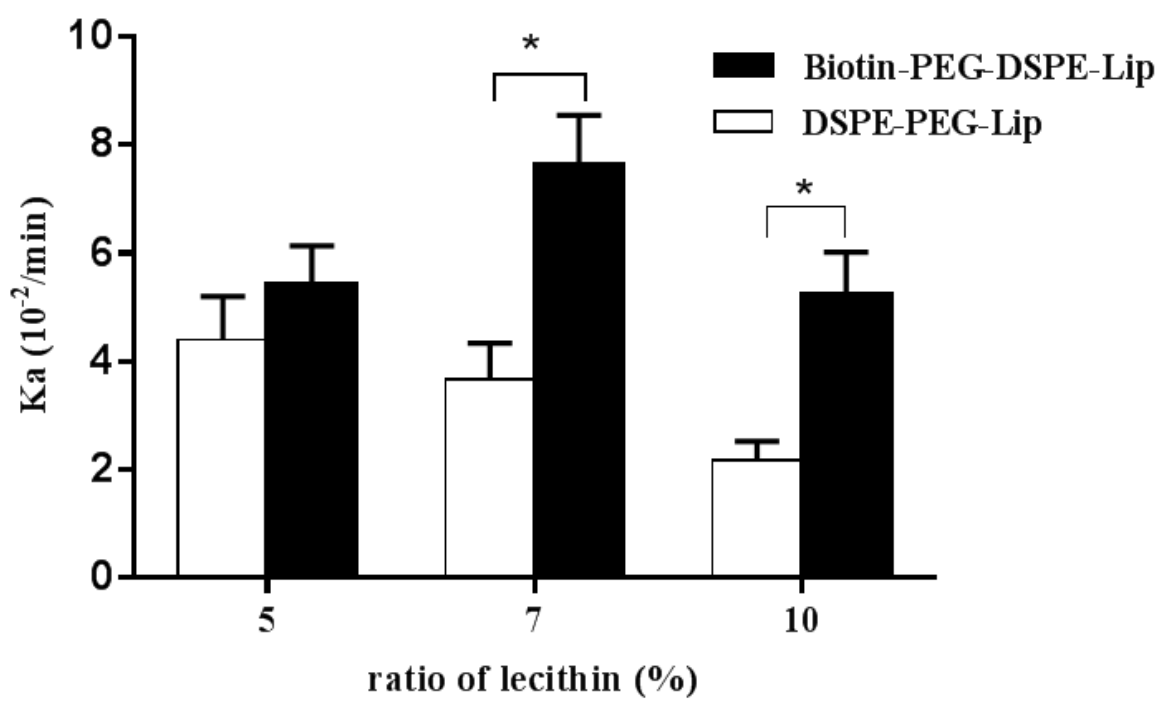

Figure 3: Rat single-pass intestinal perfusion test on DSPE-PEG-Lip and Biotin-PEG-DSPE-Lip with various PEG and Biotin ratios. $\mathrm{P} \leq 0.05:^{*}$

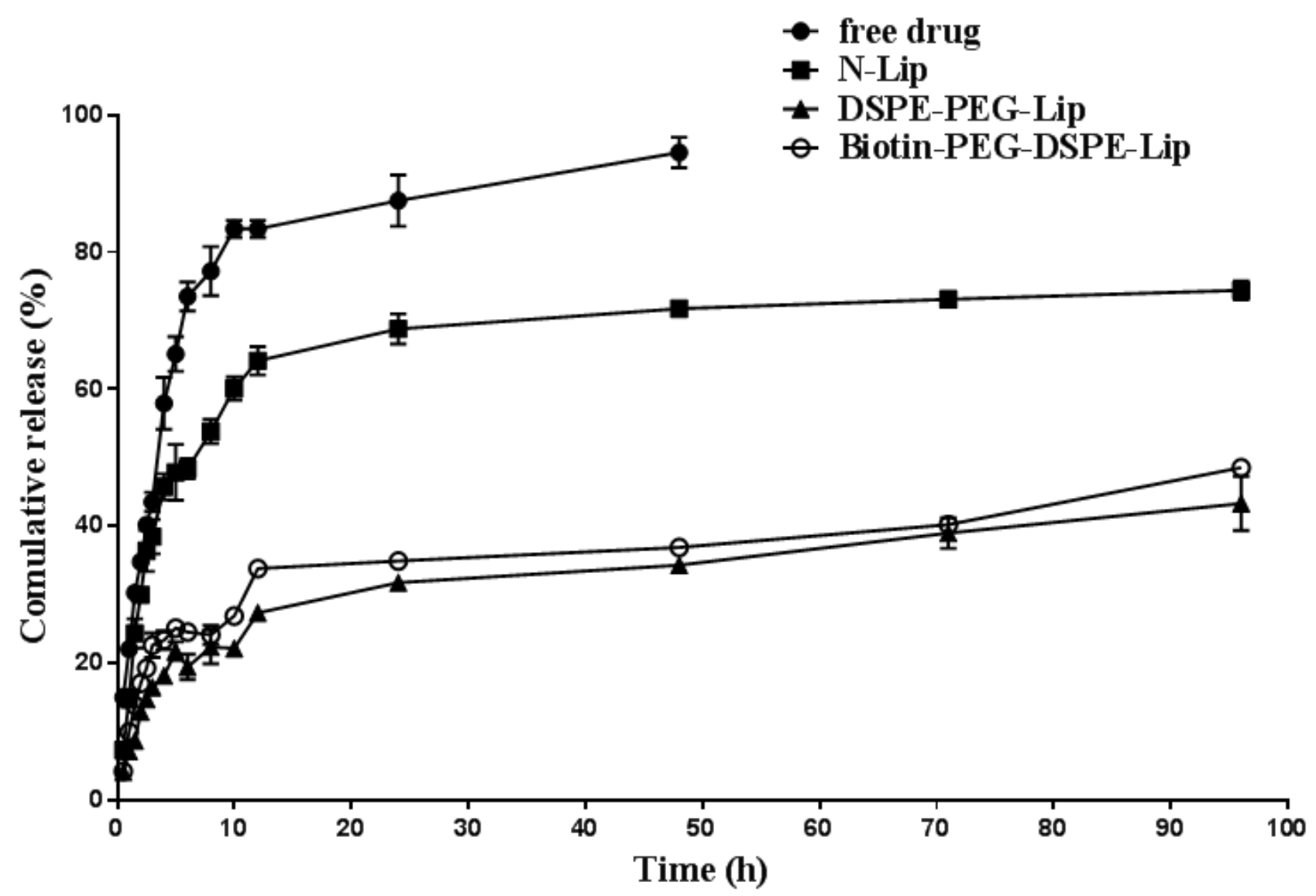

Figure 4: The in vitro release of free drug and Cur liposomes within $96 \mathrm{~h}$.

from a human colon adenocarcinoma, is an ideal cellular model in research of oral drugs. The cells exhibit many of the functional and morphological properties of the human intestinal enterocytes. After cultured for nearly 21 days, cells would differen- tiate into small intestinal microvilli, which resulted in the monolayer much tighter and secretion of alkaline phosphatase (ALP/AKP). This dense cellular monolayer could be implied as intestinal wall to investigate the absorption mechanism of curcumin 


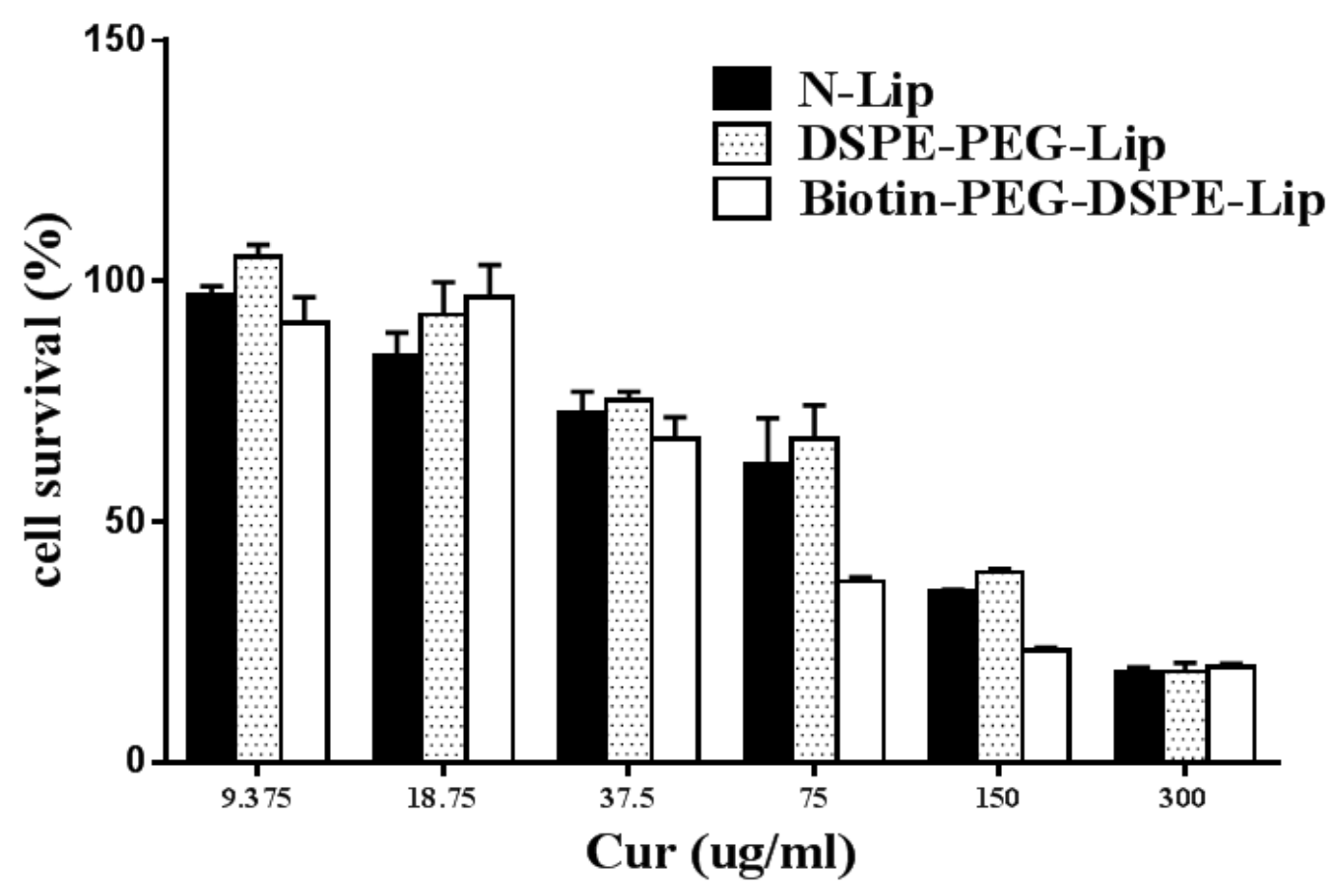

Figure 5: MTT assay results of the curcumin liposomes on cell survival.

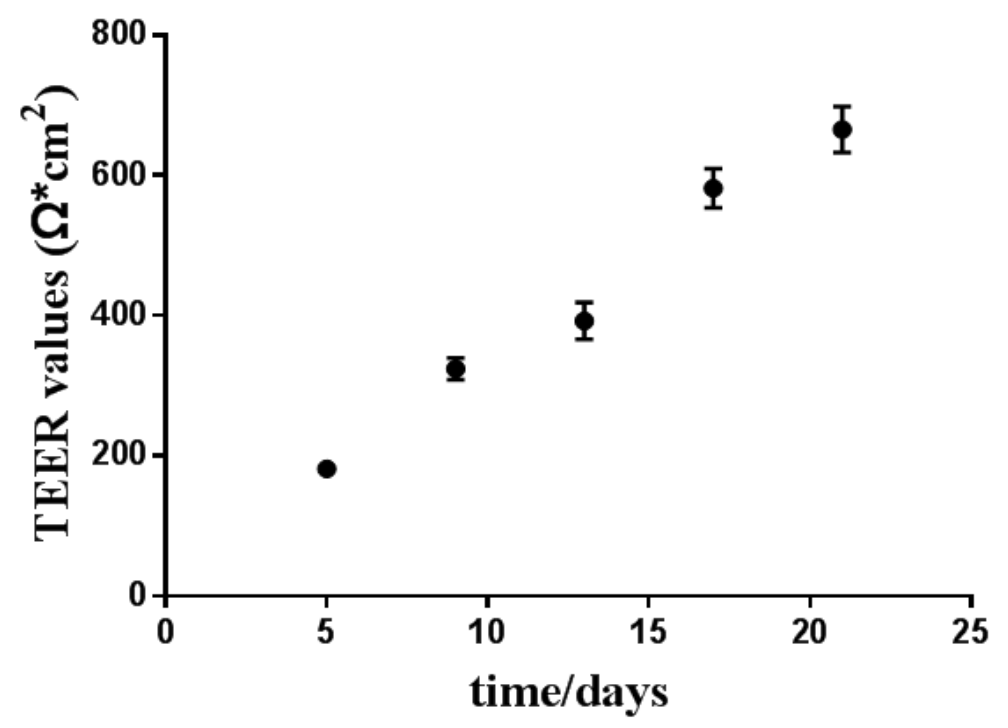

Figure 6: The TEER values of Caco-2 cells measured in 21 days.

liposomes. We used TEER values and ALP/AKP kits to check whether the monolayer had established. As the Figure 5 shows, the TEER value had reached to $600 \Omega^{*} \mathrm{~cm}^{2}$ in 21 days, it revealed that the monolayer was tight enough to be used. Otherwise, the value of ALP/AKP in AP sides suggested the differentiation of small intestinal microvilli, as the Table 3 shows, the values had been more than triple in 21 days, which indicated the microvilli had grown.

Permeability assessment of the Cur liposomes: The solubility and stability of curcumin in liposomes
Table 3: Values of ALP/AKP in 21 days.

\begin{tabular}{|l|l|l|l|}
\hline T. & 9 d & 13 d & 21 d \\
\hline AP/BP & $2.38 \pm 0.38$ & $4.82 \pm 0.57$ & $8.10 \pm 0.93$ \\
\hline
\end{tabular}

can be changed, at the same time, the permeability of curcumin were also different. Lipid bilayer can increase the transmembrane ability of curcumin, and as the Figure 6 shows that $\mathrm{N}$-Lip had the best Papp values. While caco-2 monolayer is short of mucous layer as the normal physiological environment does. The mucous layer is main barrier of oral 


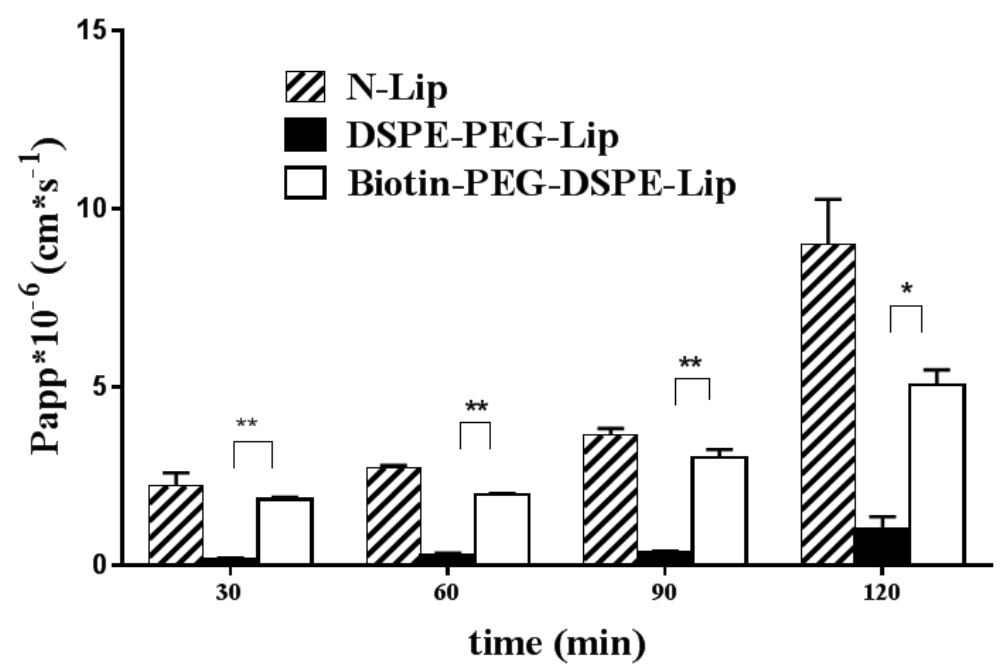

Figure 7: Permeability assessment of the Cur liposomes in $120 \mathrm{~min} . \mathrm{P} \leq 0.05:{ }^{*}, \mathrm{P} \leq 0.001:{ }^{* *}$

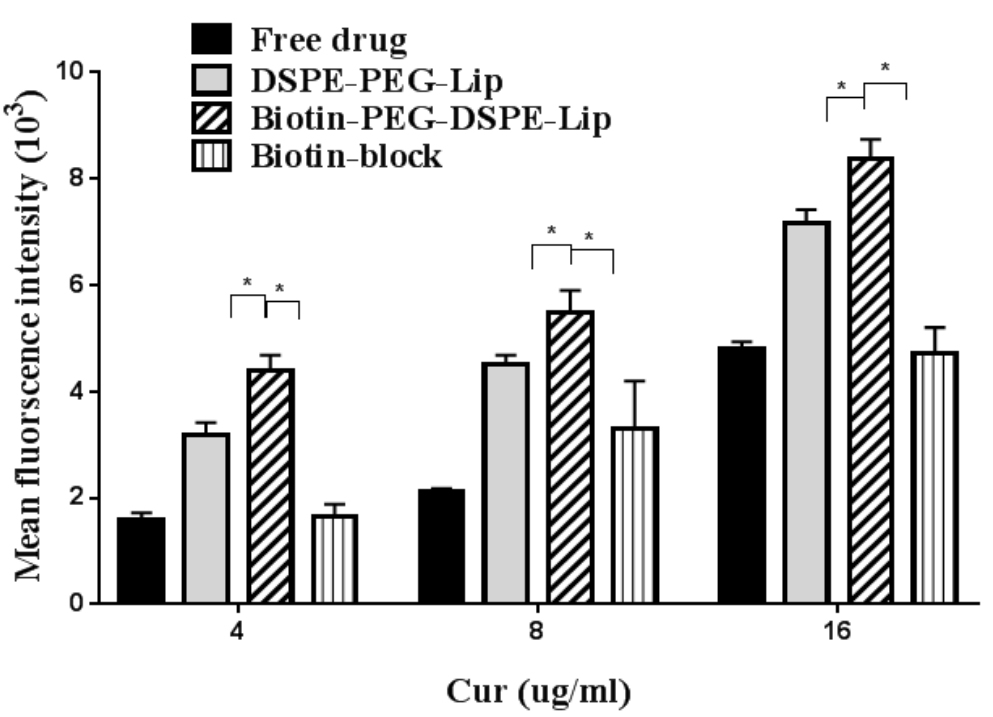

Figure 8: The uptake of caco-2 cells for different curcumin preparations. $\mathrm{P} \leq 0.05$ : *

administration drug to be absorbed. PEG is an ideal hydrophilic compound to help liposomes enter across the mucous layer, nevertheless, the Papp of DSPE-PEG-Lip were all under $1.0 \times 10^{-6} \mathrm{~cm} / \mathrm{s}$, indicating that even though PEG enhance the hydrophilic of liposomes, but decrease the cross ability of liposomes. The biotin-PEG-DSPE-Lip had a higher cross quantity than DSPE-PEG-Lip, it supposed that this kind of liposomes can be transported across the small intestinal better and more importantly finding the biotin receptors to realize the active absorption by targeting protein.

Uptake study: There are many ways for drugs and preparations to across the monolayer and intestinal tract, including simple diffusion and active transportation. While for macromolecular substanc- es such as liposomes, endocytosis is the main pathway to cross into cells. Compared to free drug and undecorated liposomes, cellular uptake study demonstrated that biotin decorated ones could be more into the caco- 2 cells (Figure 7). The addition of biotin-PEG-DSPE increased the uptake of liposomes, which according to the biotin-block group, having significant $(P \leq 0.05)$ lower uptake than nonblock biotin group one. There are biotin receptors on the caco- 2 cells surface that pre-blocked by biotin may lead to competition for biotin-PEG-DSPELip. The receptors had been occupied by biotin, decreasing the liposomes uptake into cells. As the consequence that biotin could help cellular uptake of curcumin liposomes (Figure 8). 


\section{Conclusion}

To conclusion, this study finished the preparation of curcumin liposomes which were successfully developed to improve oral bioavailability of curcumin. Both biotin decorated and undecorated liposomes showed a mean size $123.1 \pm 0.54$ $\mathrm{nm}$. Compared to curcumin powders, this delivery demonstrated greater chemical stability in aqueous medium, curcumin water solubility, physical stability in Gl fluids and permeation across Caco-2 cells.

Drug delivery systems like liposomes have a very good prospect in pharmaceutics for poorly soluble in water and lipophilic drugs such as curcumin. While liposomes should be eliminated by the phagocytosis of macrophagocytes in body. In many studies, PEGylation can markedly prolong the retention of drug in blood, overcoming the medicinal defect. We chose PEGylation lecithin to form DSPEPEG-Lip as the contrast to ensure those liposomes had similar size and cell uptake ability with normal liposomes.

However, intestinal absorption is the key process to the bioavailability for oral drugs. Liposomes would be destroyed while crossing the intestinal tract because of the variation of $\mathrm{pH}$ value, and also would be excreted by intestinal tract movement. As many study showed that there were biotin receptors on the intestinal cells, which can be recognized by biotin. So we designed liposomes decorated by biotin, which had a good morphology and drug release. In the caco-2 cells model, Biotin-PEG-DSPELip had a great cell uptake capacity than undecorated ones. This study testified that the biotin decorated liposomes which we prepared could increase the intestinal uptake of drug.

\section{Acknowledgments}

This work has been supported by the Science and Technology Innovation Team Project of Ningbo Science and Technology Bureau, China (2017R435004), and the Key Laboratory of Ningbo, China (No. 2016A22002), National Natural Science Foundation of China (NSFC) 81773673. It also thanks to the support of project from Education Department of Zhejiang (Y201636697).

\section{References}

1. Mahran RI, Hagras MM, Sun D, Brenner DE (2017) Bringing curcumin to the clinic in cancer prevention: A review of strategies to enhance bioavailability and efficacy. AAPS J 19: 54-81.
2. Zhou S, Zhang S, Shen H, Chen W, Xu H, et al. (2017) Curcumin inhibits cancer progression through regulating expression of microRNAs. Tumour Biol 39.

3. Ullah F, Liang A, Rangel A, Gyengesi E, Niedermayer G, et al. (2017) High bioavailability curcumin: An anti-inflammatory and neurosupportive bioactive nutrient for neurodegenerative diseases characterized by chronic neuroinflammation. Arch Toxicol 91: 1623-1634.

4. Maiti P, Manna J, Veleri S, Frautschy S (2014) Molecular chaperone dysfunction in neurodegenerative diseases and effects of curcumin. Biomed Res Int 2014.

5. Liu J, Chen S, Lv L, Song L, Guo S, et al. (2013) Recent progress in studying curcumin and its nano-preparations for cancer therapy. Current Pharmaceutical Design 19: 1974-1993.

6. Dhule SS, Penfornis P, He J, Harris MR, Terry T, et al. (2014) The combined effect of encapsulating curcumin and C6 ceramide in liposomal nanoparticles against osteosarcoma. Mol Pharm 11: 417-427.

7. Mahmud M, Piwoni A, Filipczak N, Janicka M, Gubernator J (2016) Long-circulating curcumin-loaded liposome formulations with high incorporation efficiency, stability and anticancer activity towards pancreatic adenocarcinoma cell lines in vitro. PLoS One 11: e0167787.

8. Catalan-Latorre A, Ravaghi M, Manca ML, Caddeo C, Marongiu F, et al. (2016) Freeze-dried eudragit-hyaluronan multicompartment liposomes to improve the intestinal bioavailability of curcumin. Eur J Pharm Biopharm 107: 49-55.

9. Li Y, C Zhu (2017) Mechanism of hepatic targeting via oral administration of DSPE-PEG-cholic acid-modified nanoliposomes. Int J Nanomedicine 12: 16731684.

10.Zhang X, Qi J, Lu Y, He W, Li X, et al. (2014) Biotinylated liposomes as potential carriers for the oral delivery of insulin. Nanomedicine 10: 167-176.

11.Anna Bogusiewicz, Gunnar Boysen, Donald M Mock (2015) In HepG2 cells, coexisting carnitine deficiency masks important indicators of marginal biotin deficiency. J Nutr 145: 32-40.

12. Mandracchia D, Rosato A, Trapani A, Chlapanidas T, Montagner IM, et al. (2017) Design, synthesis and evaluation of biotin decorated insulin-based polymeric micelles as long-circulating nanocarriers for targeted drug delivery. Nanomedicine 13: 12451254. 
13. Derycke AS, De Witte PA (2002) Transferrin-mediated targeting of hypericin embedded in sterically stabilized PEG-liposomes. Int J Oncol 20: 181-187.

14.Nii T, Ishii $F$ (2005) Encapsulation efficiency of water-soluble and insoluble drugs in liposomes prepared by the microencapsulation vesicle method. Int J Pharm 298: 198-205.

15. Ohnishi N, Yamamoto E, Tomida H, Hyodo K, Ishihara $\mathrm{H}$, et al. (2013) Rapid determination of the encapsulation efficiency of a liposome formulation using column-switching HPLC. Int J Pharm 441: 67-74.

16.Singh G, Pai RS (2015) Trans-resveratrol self-nano-emulsifying drug delivery system (SNEDDS) with enhanced bioavailability potential: Optimization, pharmacokinetics and in situ single pass intestinal perfusion (SPIP) studies. Drug Deliv 22: 522-530.

17.Escribano E, Sala XG, Salamanca J, Navarro CR, Regué JQ (2012) Single-pass intestinal perfusion to establish the intestinal permeability of model drugs in mouse. Int J Pharm 436: 472-477.

18.Sun $\mathrm{YH}$, He $\mathrm{X}$, Yang $\mathrm{XL}$, Dong $\mathrm{CL}$, Zhang $\mathrm{CF}$, et al. (2014) Absorption characteristics of the total alkaloids from Mahonia bealei in an in situ single-pass intestinal perfusion assay. Chin J Nat Med 12: 554-560.

19.Lozoya-Agullo I, González-Álvarez I, González-Álvarez M, Merino-Sanjuán M, Bermejo M (2015) In situ perfusion model in rat colon for drug absorption studies: Comparison with small intestine and caco-2 cell model. J Pharm Sci 104: 3136-3145.

20.Scheers NM, Almgren AB, Sandberg AS (2014) Pro- posing a Caco-2/HepG2 cell model for in vitro iron absorption studies. J Nutr Biochem 25: 710-715.

21.Peng $\mathrm{Y}$, Yadava $\mathrm{P}$, Heikkinen AT, Parrott N, Railkar A (2014) Applications of a 7-day Caco-2 cell model in drug discovery and development. Eur J Pharm Sci 56: 120-130.

22.Sun $\mathrm{S}$, Zhang $\mathrm{H}$, Sun $\mathrm{F}$, Zhao L, Zhong $\mathrm{Y}$, et al. (2014) Intestinal transport of sophocarpine across the Caco2 cell monolayer model and quantification by LC/MS. Biomed Chromatogr 28: 885-890.

23.Zheng M, Zhou H, Wan H, Chen YL, He Y (2015) Effects of herbal drugs in Mahuang decoction and their main components on intestinal transport characteristics of Ephedra alkaloids evaluated by a Caco- 2 cell monolayer model. J Ethnopharmacol 164: 22-29.

24.Morisaki T, Hou XL, Takahashi K, Takahashi K (2013) Baicalin pharmacokinetic profile of absorption process using novel in-vitro model: Cytochrome P450 3A4-induced Caco-2 cell monolayers combined with rat intestinal rinse fluids. J Pharm Pharmacol 65: 1526-1535.

25.Huang XZ, Li ZR, Zhu LB, Huang HY, Hou LL, et al. (2014) Inhibition of p38 mitogen-activated protein kinase attenuates butyrate-induced intestinal barrier impairment in a Caco-2 cell monolayer model. J Pediatr Gastroenterol Nutr 59: 264-269.

26. Hubatsch I, Ragnarsson EG, Artursson P (2007) Determination of drug permeability and prediction of drug absorption in Caco-2 monolayers. Nat Protoc 2: 2111-2119. 